\title{
Calibration of MEMS Accelerometers and Magnetometers for Rotor UAV Attitude Estimation
}

\author{
Xiang Ren \\ Dept. of Mechanical Engineering and Automation \\ Harbin Institute of Technology Shenzhen Graduate \\ School \\ Shenzhen, China \\ e-mail: jn_renxiang@126.com
}

\author{
Shoubin Liu* \\ Dept. of Mechanical Engineering and Automation \\ Harbin Institute of Technology Shenzhen Graduate \\ School \\ Shenzhen, China \\ *Corresponding author: mesbliu@hitsz.edu.cn
}

\begin{abstract}
Rotor unmanned aerial vehicles (UAV) usually adopt MEMS gyros, accelerometers and magnetometers to determine its navigation attitude. Because a MEMS gyro has a drawback of angle drift, its attitude data is often corrected by the data solved with accelerometers and magnetometers. This paper presents a static and scalar calibration method for accurate solving of attitude angles with MEMS triaxial accelerometers and triaxial magnetometers. Based on the facts that vector sum of triaxial outputs of accelerometers equals to the gravity acceleration, and vector sum of triaxial outputs of magnetometers equals to the geomagnetic vector, the error equations are established. Taking the sum of error squares as the objective function, a nonlinear least square method is applied to solve the optimal calibration parameters. A Kalman filter is used to suppress the random error of output signals of sensors. A precise triaxial turn table is used to vary the spatial attitude of the sensor module for data sampling. Output signals of sensors at 32 different attitudes are captured, and unknown calibration parameters are solved. It is found that the variation of the difference values between the attitude angles calculated with the calibrated parameters and the attitude angles indicated by triaxial turn table is around $\pm 1^{\circ}$. It is proved that the proposed calibration method for MEMS triaxial accelerometers and triaxial magnetometers is accurate and feasible.
\end{abstract}

Keywords-Attitude Estimation; MEMS; Accelerometers; Magnetometers; Calibration

\section{INTRODUCTION}

Rotor unmanned aerial vehicles (UAV) usually adopt MEMS gyros, accelerometers and magnetometers to determine its navigation attitude. The drift of highprecision gyros is around $10^{-4} \mathrm{o} / \mathrm{h}$, while the drift of MEMS gyros is typically $10^{-1}-10^{-2} \mathrm{o} / \mathrm{h}$. Because a MEMS gyro has a large drift, its attitude data after long hours is not reliable. And its attitude data is often corrected by the data solved with MEMS accelerometers and magnetometers. Data fusion algorithms, such as extended Kalman filter (EKF) fusion, cross product fusion and complementary filter fusion are often used in attitude correction [1-4].

This paper addresses a problem of solving attitude angles with MEMS triaxial accelerometers and triaxial magnetometers. To achieve accurate attitude data, calibration parameters of accelerometers and magnetometers are determined using a static and scalar calibration method $[5,6]$. A miniature sensor module, composing MEMS triaxial accelerometers and triaxial magnetometers, is fixed on a precise triaxial turn table. By varying spatial attitude of the turn table, output signals of the miniature sensor module at 32 different attitudes are captured. After Kalman filtering, average sample data is input to two calibration models of accelerometers and magnetometers. Based on the facts that vector sum of triaxial outputs of accelerometers equals to the gravity acceleration, and vector sum of triaxial outputs of magnetometers equals to the geomagnetic vector, the error equations of accelerometers and magnetometers are established. Taking the sum of error square as the objective function, a nonlinear least square method is applied to solve the optimal calibration parameters. The proposed calibration method is further proved by nine sets of measurements. Difference values between the attitude angles calculated with the calibrated parameters and the attitude angles indicated by triaxial turn table are calculated, and the variation is analyzed.

\section{KALMAN FILTERING OF OUTPUT SignALS}

As shown in Fig. 1(a), an output signal of the miniature sensor module at the moment $k$, can be expressed by

$$
z_{k}=x_{k}+v_{k}
$$

where $x_{k}$ is the filtered signal at the moment $k$, which can be expressed by

$$
x_{k}=x_{k-1}+w_{k-1}
$$

where $v_{k}$ and $w_{k}$ are two Gaussian-distributed white noise sequences with zero mean value.

Based on (1) and (2), the recursion formula of Kalman filter is given by

$$
\left.\begin{array}{l}
\hat{X}_{k / k-1}=\hat{X}_{k-1 / k-1} \\
P_{k / k-1}=P_{k-1 / k-1}+\mathrm{Q} \\
K_{k / k}=P_{k / k-1} /\left(P_{k / k-1}+\mathrm{R}\right) \\
\hat{X}_{k / k}=\hat{X}_{k / k-1}+K_{k / k}\left(Z_{k}-\hat{X}_{k / k-1}\right) \\
P_{k / k}=\left(I-K_{k / k}\right) P_{k / k-1}
\end{array}\right\}
$$


with initial values of $\mathrm{Q}=0.0001, X_{k / k-1}=9.8$ and $P_{k / k-I}=1$.

In (3), the value of $\mathrm{R}$ can affect the suppression of high-frequency components. With the value of $\mathrm{R}$ increasing, the suppression will increase. Fig. 1(b) shows the filtered signal with $\mathrm{R}=1$.

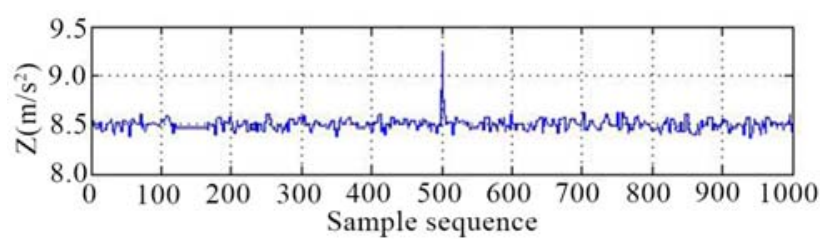

(a)

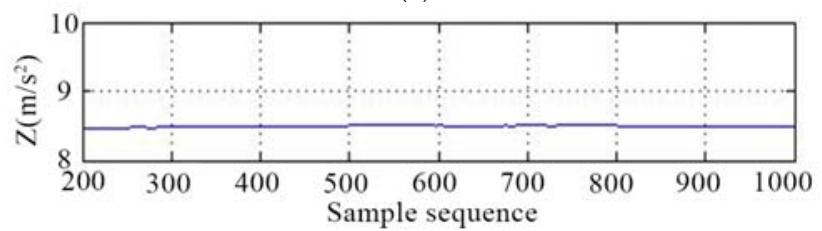

(b)

Figure 1. An output signal of the miniature sensor module (a), and the filtered signal with $\mathrm{R}=1$ (b)

\section{MATHEMATICAL MODELS}

The acceleration vector of triaxial accelerometers can be expressed by $[5,7]$

$$
A=S(V-O)=\left[\begin{array}{ccc}
S_{x x} & S_{x y} & S_{x z} \\
S_{y x} & S_{y y} & S_{y z} \\
S_{z x} & S_{z y} & S_{z z}
\end{array}\right]\left[\begin{array}{c}
V_{x}-O_{x} \\
V_{y}-O_{y} \\
V_{z}-O_{z}
\end{array}\right]=\left[\begin{array}{c}
a_{x} \\
a_{y} \\
a_{z}
\end{array}\right]
$$

where $V^{T}=\left[V_{x}, V_{y}, V_{z}\right]$ is output vector of MEMS triaxial accelerometers, $A^{T}=\left[a_{x}, a_{y}, a_{z}\right]$ is the actual acceleration vector composing three components of the vector of gravity acceleration in $\mathrm{x}, \mathrm{y}, \mathrm{z}$ axes, $O^{T}=\left[O_{x}, O_{y}, O_{z}\right]$ is the bias vector. $\mathrm{S}$ is the scale factor matrix, where $S_{x x}, S_{y y}, S_{z z}$, are scale coefficients of three axes, and $S_{x y}, S_{x z}, S_{y x}, S_{y z}, S_{z x}$, $S_{z y}$ are cross-axis coefficients.

Formula (4) is the 12-parameter calibration model of accelerometers. Because cross-axis coefficients are small values and can be neglected, Formula (4) can be simplified as

$$
\left.\begin{array}{l}
a_{x}=S_{x x}\left(V_{x}-O_{x}\right) \\
a_{y}=S_{y y}\left(V_{y}-O_{y}\right) \\
a_{z}=S_{z z}\left(V_{z}-O_{z}\right)
\end{array}\right\}
$$

Formula (5) is the 6-parameter calibration model of accelerometers. Similarly, the 6-parameter calibration model of magnetometers can be expressed by

$$
\left.\begin{array}{l}
h_{x}=T_{x x}\left(H_{x}-Q_{x}\right) \\
h_{y}=T_{y y}\left(H_{y}-Q_{y}\right) \\
h_{z}=T_{z z}\left(H_{z}-Q_{z}\right)
\end{array}\right\}
$$

where $Q^{T}=\left[Q_{x}, Q_{y}, Q_{z}\right]$ is magnetic compass deviation vector, $T_{x x}, T_{y y}, T_{z z}$, are scale coefficients of three axes, $H_{b}{ }^{T}=\left[h_{x}, h_{y}, h_{z}\right]$ is the actual geomagnetism vector composing three components of the geomagnetic vector in $\mathrm{x}, \mathrm{y}, \mathrm{z}$ axes of the body coordinate system. Formula (6) is the 6-parameter calibration model of magnetometers.

Pitch angle $\theta$ and roll angle $\varphi$ can be calculated with calibrated acceleration vector $A^{T}=\left[a_{x}, a_{y}, a_{z}\right]$, and yaw angle $\Psi$ can be determined with calibrated geomagnetism vector $H_{b}{ }^{T}=\left[h_{x}, h_{y}, h_{z}\right]$ in the horizontal plane. Because the tilt of magnetometers can affect the solution of yaw angle $\Psi$, pitch angle $\theta$ and roll angle $\varphi$ are involved in the calculation of yaw angle $\Psi$ for compensating the tilt error $[8,9]$.

As shown in Fig. 2(b), defining a body coordinate system $O_{b}-X_{b} Y_{b} Z_{b}$ with $X_{b}$ indicating the front, $Y_{b}$ indicating the right, and $Z_{b}$ indicating the bottom, a navigation coordinate system $O_{n}-X_{n} Y_{n} Z_{n}$ with $X_{n}$ indicating the North, $Y_{n}$ indicating the East, and $Z_{n}$ indicating the ground, and a rotation matrix $C_{b}{ }^{n}$ following Z-Y-X, the geomagnetism vector in the navigation coordinate system $O_{n}-X_{n} Y_{n} Z_{n}$ can be expressed by

$$
H_{n}=C_{b}^{n} \bullet H_{b}=\left(\begin{array}{ccc}
\cos \theta & \sin \varphi \sin \theta & \cos \varphi \sin \theta \\
0 & \cos \varphi & -\sin \varphi \\
-\sin \theta & \sin \varphi \cos \theta & \cos \varphi \cos \theta
\end{array}\right)\left(\begin{array}{l}
h_{x} \\
h_{y} \\
h_{z}
\end{array}\right)
$$

where $H_{n}{ }^{T}=\left[h_{x}{ }^{n}, h_{y}{ }^{n}, h_{z}{ }^{n}\right]$ is the geomagnetism vector composing three components in $\mathrm{x}, \mathrm{y}, \mathrm{z}$ axes of the navigation coordinate system. Attitude angles, $\theta, \varphi$ and $\Psi$ can be calculated by

$$
\left.\begin{array}{l}
\theta=-\arctan 2\left(\frac{a_{x}}{\sqrt{a_{y}{ }^{2}+a_{z}^{2}}}\right) \\
\varphi=\arctan 2\left(\frac{a_{y}}{a_{z}}\right) \\
\Psi=\arctan 2\left(\frac{h_{y n}}{h_{x n}}\right)+\beta_{m}
\end{array}\right\}
$$

where $\beta_{m}$ is the magnetic declination which can be calculated with the world geomagnetic model (WMM) provided by the National Oceanic and Atmospheric Administration (NOAA), and $\theta, \varphi, \Psi$ are pitch angle, roll angle and yaw angle.

\section{DETERMINATION OF CALIBRATION PARAMETERS}

Six calibration parameters of acceleration in (5) can be solved simply with six special attitudes, i.e. $[\theta, \varphi, \Psi]=[0,0$, $0],[\pi / 2,0,0],[-\pi / 2,0,0],[0, \pi / 2,0],[0,-\pi / 2,0],[0,-\pi, 0$,$] .$ Six calibration parameters of geomagnetism in (6) can also be solved with the similar method. The accuracy of sixposition calibration method depends on how well one of the axes of the body coordinate system is aligned with the direction of the vector of the gravity acceleration. To achieve more accurate calibration parameters, more than six attitudes can be used $[5,10]$.

Based on the facts that vector sum of triaxial outputs of accelerometers equals to the gravity acceleration, and vector sum of triaxial outputs of magnetometers equals to the geomagnetic vector, the measurement equations of acceleration and geomagnetism are given by

$$
\left.\begin{array}{l}
a_{x}^{2}+a_{y}^{2}+a_{z}^{2}=g^{2} \\
h_{x}^{2}+h_{y}^{2}+h_{z}^{2}=H_{0}^{2}
\end{array}\right\}
$$

In terms of (5), (6) and (9), error equations of acceleration and geomagnetism can be obtained as 


$$
\left.\begin{array}{l}
r_{a i}=\sum_{i=x, y, z}\left\{\left[S_{i i}\left(V_{i}-O_{i}\right)\right]^{2}\right\}-g^{2} \\
r_{h j}=\sum_{j=x, y, z}\left\{\left[T_{j j}\left(H_{j}-Q_{j}\right)\right]^{2}\right\}-H_{0}^{2}
\end{array}\right\}
$$

Taking the sum of square errors of $m$ measurements as the objective function, objective functions of acceleration and geomagnetism can be expressed as

$$
\left.\begin{array}{l}
E_{a}=E_{a}\left(O_{x}, O_{y}, O_{z}, S_{x x}, S_{y y}, S_{z z}\right)=\frac{1}{2} \sum_{i=1}^{m} r_{a i}^{2} \\
E_{h}=E_{h}\left(Q_{x}, Q_{y}, Q_{z}, T_{x x}, T_{y y}, T_{z z}\right)=\frac{1}{2} \sum_{j=1}^{n} r_{h j}^{2}
\end{array}\right\}
$$

where $1 / 2$ is a fixed coefficient for simplifying the gradient expression. To minimize (11), the gradient value of $E_{a}$ or $E_{h}$ should be zero, i.e.

$$
\nabla E(X)=\nabla\left(\frac{1}{2} r(x)^{T} r(x)\right)=r(x)^{T} J=0
$$

where $r(x)=\left[r_{1}, r_{2}, \ldots, r_{m}\right]^{\mathrm{T}}$ is the error vector of acceleration or geomagnetism, $\mathrm{J}$ is the Jacobian matrix of $r(x)$. Formula (12) expresses multi-variable nonlinear equations, which can be solved by Levenberg-Marquardt (LM) method [11, 12]. The LM iteration formula is given by

$$
x^{n+1}=x^{n}-\left(J^{T} J+\lambda \operatorname{diag}\left(J^{T} J\right)\right)^{-1} J^{T} r\left(x^{n}\right)
$$

where $x^{n}$ indicates the $n$-th iteration of the calibration parameter vector of acceleration or geomagnetism, i.e.

$$
\left.\begin{array}{rl}
x_{a}^{n} & =\left[O_{x}^{n}, O_{y}^{n}, O_{z}^{n}, S_{x x}^{n}, S_{y y}^{n}, S_{z z}^{n}\right] \\
x_{h}^{n} & =\left[Q_{x}^{n}, Q_{y}^{n}, Q_{z}^{n}, T_{x x}^{n}, T_{y y}^{n}, T_{z z}^{n}\right]
\end{array}\right\}
$$

$\lambda$ is a damping parameter varied at each iteration for enhancing convergence and extending the range of initial values. Iterations are terminated when $x^{n+1}$ satisfies the criterion as

$$
\left\|x^{n+1}-x^{n}\right\|<\varepsilon
$$

After the termination of LM iteration, total twelve calibration parameters of acceleration and geomagnetism can be determined.

\section{EXPERIMENT}

Fig. 2(a) shows a miniature sensor module, composing two MEMS chips, MPU6050 and HMC5883L. The triaxial accelerometers integrated in MPU6050, and the triaxial magnetometers integrated in HMC5883L, are attitude sensors to be calibrated. The miniature sensor module is fixed on a precise triaxial turn table, as shown in Fig. 2(b). The triaxial turn table is composed of a small $\Psi$-axis turn table (KSP-406M by Sigma koki), and a small $\theta-\varphi$ doubleaxis turn table (GOH-40B35 by Sigma koki). By varying individual angles of the triaxial turn table, the miniature sensor module is set at 32 different spatial attitudes. Static output signals of two kinds of sensors at different attitudes are captured by DSP sampling system.

Six signals are transferred to a computer via DSP sampling system. Three signals of triaxial accelerometers are Kalman-filtered due to the presence of serious noises, and three signals of triaxial magnetometers are non-filtered due to the stability of signals. An average of 1000 measurements is taken as the output at a sampling attitude. These average data is input to (10) to generate individual error components at 32 different attitudes. In terms of (11), squares of error components are accumulated to form the objective functions. By using LM iteration formula (13), total 12 calibration parameters are obtained, as shown in Tables I and II.

To verify the 12 calibration parameters of the sensors, attitude angles are calculated in terms of (8) and the calibrated parameters of Tables I and II. By comparing the calculated attitude angles with those indicated by the triaxial turn table, difference values are obtained. The variation of difference values can be further calculated.

Fixing pitch angle $\theta=15^{\circ}$ and yaw angle $\Psi=0^{\circ}$, and changing roll angle $\varphi$ from $-20^{\circ}$ to $+20^{\circ}$ with a step of $5^{\circ}, 9$ sets of measurements of triaxial accelerometers are obtained. 9 sets of roll angle $\varphi$ are calculated with the average sampling data and six calibrated parameters of acceleration. The difference values relative to those of turn table can be obtained, as shown in Table III.

Fixing pitch angle $\theta=15^{\circ}$ and roll angle $\varphi=20^{\circ}$, and changing yaw angle $\Psi$ from $0^{\circ}$ to $360^{\circ}$ with a step of $45^{\circ}, 9$ sets of measurements of triaxial geomagnetism are obtained. 9 sets of yaw angle $\Psi$ are calculated with the average sampling data and six calibrated parameters of geomagnetism. The difference values relative to those of turn table can be obtained, as shown in Table IV.
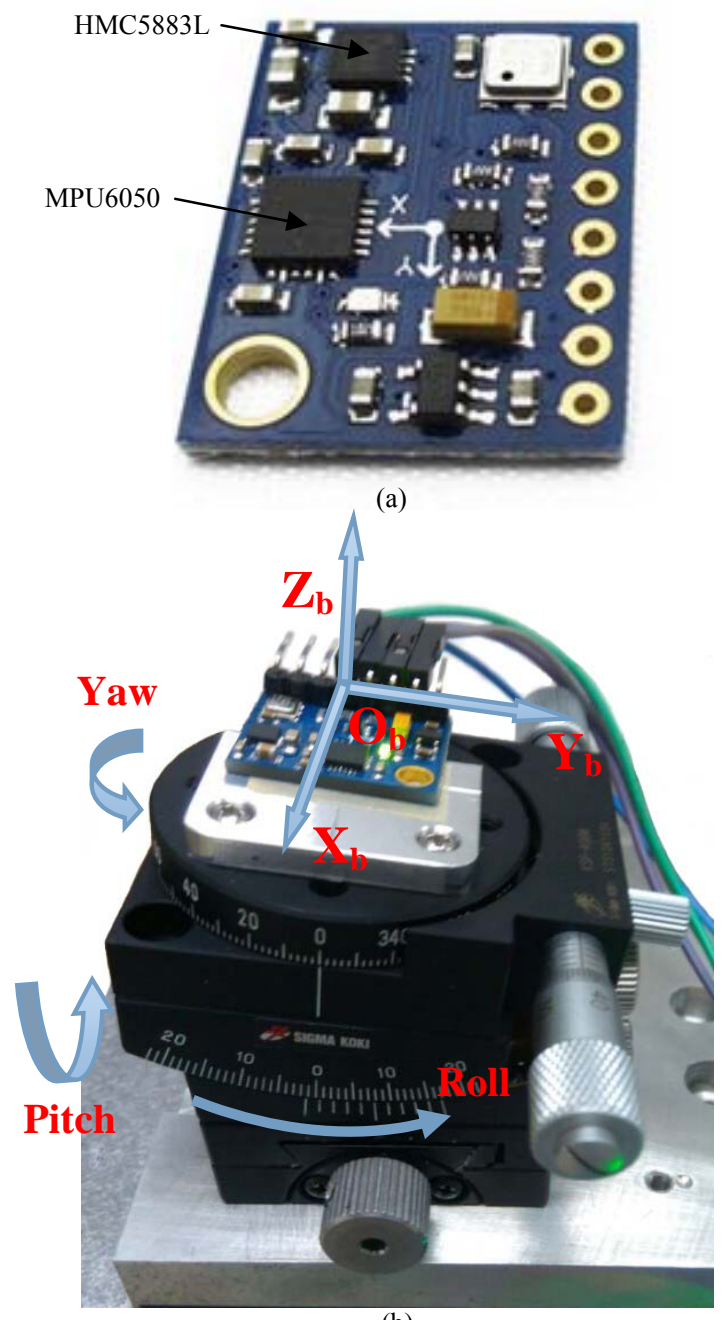

(b)

Figure 2. The miniature sensor module (a), and the precise triaxial turn table for varying attitude angles (b) 
TABLE I. CALIBRATED PARAMETERS OF THE TRIAXIAL ACCELEROMETERS

\begin{tabular}{|l|c|c|c|}
\hline Item & $\mathbf{X}$ & $\mathbf{Y}$ & $\mathbf{Z}$ \\
\hline Scale factor & 1.0036 & 1.0019 & 0.9992 \\
\hline Bias $\left(\mathbf{m . s}^{-2}\right)$ & $0.016 \mathrm{~g}$ & $0.0017 \mathrm{~g}$ & $0.0013 \mathrm{~g}$ \\
\hline
\end{tabular}

TABLE II. CALIBRATED PARAMETERS OF THE TRIAXIAL MAGNETOMETERS

\begin{tabular}{|l|c|c|c|}
\hline Item & $\mathbf{X}$ & $\mathbf{Y}$ & $\mathbf{Z}$ \\
\hline Scale factor & 0.9647 & 0.9715 & 1.1232 \\
\hline Bias(Gauss) & 0.0007 & 0.0139 & -0.1121 \\
\hline
\end{tabular}

TABLE III. THE ROLL ANGLES CALCULATED WITH 6 CALIBRATED PARAMETERS OF ACCELERATION AND DIFFERENCE VALUES RELATIVE TO THOSE OF TURN TABLE

\begin{tabular}{|l|c|c|c|c|}
\hline Roll( $\left(^{\circ}\right)$ & -20 & -15 & -10 & -5 \\
\hline $\begin{array}{l}\text { Before } \\
\text { calibration }\end{array}$ & -19.56 & -14.71 & -9.69 & -4.76 \\
\hline $\begin{array}{l}\text { After } \\
\text { calibration }\end{array}$ & -19.72 & -14.87 & -9.83 & -4.88 \\
\hline Difference & 0.28 & 0.13 & 0.17 & 0.12 \\
\hline
\end{tabular}

\begin{tabular}{|c|c|c|c|c|}
\hline 0 & 5 & 10 & 15 & 20 \\
\hline 0.26 & 5.28 & 10.33 & 15.20 & 20.13 \\
\hline 0.16 & 5.20 & 10.27 & 15.17 & 20.11 \\
\hline 0.16 & 0.20 & 0.27 & 0.17 & 0.11 \\
\hline
\end{tabular}

TABLE IV. THE YAW ANGLES CALCULATED WITH 6 CALIBRATED PARAMETERS OF GEOMAGNETISM AND DIFFERENCE VALUES RELATIVE TO THOSE OF TURN TABLE

\begin{tabular}{|c|c|c|c|c|}
\hline $\operatorname{Yaw}\left({ }^{\circ}\right)$ & 0 & 45 & 90 & 135 \\
\hline $\begin{array}{l}\text { Before } \\
\text { calibration }\end{array}$ & 20.63 & 60.6 & 106.69 & 158.86 \\
\hline $\begin{array}{l}\text { After } \\
\text { calibration }\end{array}$ & 23.11 & 67.7 & 113.32 & 158.43 \\
\hline Difference & 23.11 & 22.7 & 23.32 & 23.43 \\
\hline
\end{tabular}

\begin{tabular}{|c|c|c|c|c|}
\hline 180 & 225 & 270 & 315 & 360 \\
\hline 204.94 & 251.07 & 293.6 & 333.1 & 16.28 \\
\hline 202.46 & 247.53 & 292.97 & 337.67 & 22.54 \\
\hline 22.46 & 22.53 & 22.97 & 22.67 & 22.54 \\
\hline
\end{tabular}

In Table III, the difference values exhibit a standard deviation of $\sigma_{\text {roll }}=0.06^{\circ}$, and an average of $0.18^{\circ}$, i.e. all difference values between the calculated roll angles with calibrated parameters and those indicated by the triaxial turn table are in the range of $0.18 \pm 0.18^{\circ}$. The average value of $0.18^{\circ}$ stands for an initial tilt angle of the turn-table and sensor-module system. And the variation of $\pm 0.18^{\circ}$ stands for the calibration precision or uncertainty of accelerometers. As a contrast, the variation of difference values before calibration is $\pm 0.26^{\circ}$.

In Table IV, the difference values exhibit a standard deviation of $\sigma_{\text {yaw }}=0.36^{\circ}$, and an average of $22.86^{\circ}$, i.e. all difference values between the calculated yaw angles with calibrated parameters and those indicated by the triaxial turn table are in the range of $22.86 \pm 1.08^{\circ}$. The average value of $22.86^{\circ}$ stands for an initial magnetic declination of the turn-table and sensor-module system related to the
North. And the variation of $\pm 1.08^{\circ}$ stands for the calibration precision or uncertainty of magnetometers. As a contrast, the variation of difference values before calibration is $\pm 12.26^{\circ}$.

\section{CONCLUSION}

A static and scalar calibration method is proposed based on two similar calibration models developed for triaxial accelerometers and triaxial magnetometers. The calibration parameters, i.e. the bias and scale factors are solved with LM method by use of vector sum of triaxial outputs of accelerometers equaling to the gravity acceleration, and vector sum of triaxial outputs of magnetometers equaling to the geomagnetic vector. This method is less cost as it makes use of multi-attitude sampling data based on a triaxial turn table. Experiments show that the variation of the difference values between the attitude angles calculated with the calibrated parameters and the attitude angles indicated by triaxial turn table is around $\pm 1^{\circ}$.

\section{REFERENCES}

[1] D. Jurman, M. Jankovec, R. Kamnik, et al., "Calibration and data fusion solution for the miniature attitude and heading reference system," Sensors \& Actuators, A: Physical, vol. 138(2), 2007, pp.411-420.

[2] Y. C. Lai, S. S. Jan, and F. B. Hsiao, "Development of a low-cost attitude and heading reference system using a three-axis rotating platform". Sensors, vol. 10(4), 2010, pp. 2472-2491.

[3] T. Yoo, "Gain-scheduled complementary filter design for a MEMS based attitude and heading reference system," Sensors, vol. 11(4), 2011, pp. 3816-3830.

[4] D. Ma, J. Shiau, I. Wang, et al., "Attitude Determination Using a MEMS-Based Flight Information Measurement Unit," Sensors, vol. 12(1), 2011, pp. 1-23.

[5] I. Frosio, F. Pedersini, and N. A. Borghese, "Autocalibration of MEMS accelerometers," IEEE Transactions on Instrumentation and Measurement, vol. 58(6), 2009, pp. 2034-2041.

[6] K. Mohamadabadi, M. Hillion, "An Automated Indoor Scalar Calibration Method for Three-Axis Vector Magnetometers," IEEE Sensors Journal, vol. 14(9), 2014, pp. 3076-3083.

[7] G. W. Barber and A. S. Arrott, "History and magnetics of compass adjusting," IEEE Transactions on Magnetics, vol. 24(6), 1988, pp. 2883-2885.

[8] J. Vcelak, et al., "Errors of AMR compass and methods of their compensation," Sensors \& Actuators, A: Physical, vol. 129(1), 2006, pp. 53-57.

[9] R. Smith, A. Frost, and P. Probert, "A Sensor System for the Navigation of an Underwater Vehicle," International Journal of Robotics Research, vol. 18(7), 1999, pp. 697-710.

[10] Z. Syed, et al., "A new multi-position calibration method for MEMS inertial navigation systems," Measurement Science \& Technology, 18(7), 2007, pp. 1897-1907.

[11] D. Bates, and D. Watts, Nonlinear Regression and Its Applications, New York: Wiley, 1988.

[12] C. Ma and L. Jiang, "Some research on Levenberg-Marquardt method for the nonlinear equations," Applied Mathematics \& Computation, vol. 184(2), 2007, pp. 1032-1040. 